\title{
CURVA DE COCCIÓN DE LA ARCILLA EN LA LADRILLERA EL RECREO
}

\section{CURL OF COOKING OF THE CLAY IN THE LADRILLERA EL RECREO}

\author{
MSc. Gustavo Guerrero Gómez*, MSc. Edwin Espinel Blanco**, \\ MSc. Nelson Escobar Mora*** \\ * Universidad Francisco de Paula Santander Seccional Ocaña, Grupo de investigación en \\ tecnología y desarrollo en ingeniería GITYD, vía Acolsure sede el Algodonal Ocaña \\ Norte de Santander, Ocaña, Norte de Santander. Colombia. \\ 0375690088 ext. 210. \\ gguerrerog@ufpso.edu.co \\ ** Universidad Francisco de Paula Santander Seccional Ocaña, Grupo de investigación en \\ tecnología y desarrollo en ingeniería GITYD, vía Acolsure sede el Algodonal Ocaña \\ Norte de Santander, Ocaña, Norte de Santander. Colombia. \\ 0375690088 ext. 210. \\ eeespinelb@ufpso.edu.co. \\ **** Universidad Pontificia Bolivariana Medellín, Grupo de investigación Bioingeniería \\ GIBIOING, Cq 1 \# 70-01 Medellín, Antioquía. Colombia. \\ 4488388 ext. 14072. \\ Nelson.escobar@upb.edu.co.
}

Resumen: En un chircal artesanal que fabrica ladrillos macizos de arcilla ubicado en el municipio de Ocaña, Departamento Norte de Santander, se realizó un estudio de las temperaturas presentadas en el horno circular durante la cocción del ladrillo con sus propiedades finales, se diseñó un sistema para registrar la temperaturas en el horno utilizando el software Labview y se realizó la comparación de la curva teórica de temperaturas establecida por Munier, en Chaleur et Industrie al utilizar un análisis termicodilatométrico a la arcilla con la curva de cocción real de la arcilla obtenida en la adquisición de temperatura en el proceso de cocción de la arcilla en la ladrillera el recreo.

Palabras clave: Adquisición de datos, Curva cocción, arcilla, ladrillos.

\begin{abstract}
In a craft chircal which manufactures solid bricks clay located in the municipality of Ocaña, Norte de Santander Department, a study of the temperatures presented in the round during cooking brick with its final properties oven it was performed, a system design recording the temperatures in the oven using the Labview software and The comparison of the theoretical temperature curve established by Munier, in Chaleur et Industrie, using a thermodilatometric analysis to the clay with the actual cooking curve of the clay obtained in the temperature acquisition in the cooking process of the clay in the El Recreo brickyard.
\end{abstract}

Keywords: Data acquisition, cooking curve, clay, bricks. 


\section{INTRODUCCIÓN}

En el municipio de Ocaña departamento Norte de Santander se ha desarrollado una industria cerámica importante en la producción de materiales para la construcción como ladrillo, bloque y teja, que de acuerdo con la clasificación hecha en el Artículo 20 de la Ley 905 de 2004, el $96.67 \%$ son empresas pequeñas que desarrollan los procesos productivos de forma artesanal, utilizando hornos de fuego dormido a cielo abierto, por su bajo costo de construcción, mantenimiento y régimen de operación intermitente, realizando la quema de combustible sobre parrilla fija y no se tienen controles sobre las proporciones de ingredientes para la producción de la mezcla de arcilla de sus productos.

El proceso de producción de materiales cerámicos comprende las etapas de extracción de la materia prima, trituración, humectación, compactación, extrusión, corte, secado, cocción y apilado. El proceso es manejado en tres medidas estratégicas: la explotación minera, la transformación de la arcilla y la comercialización del producto final. Una de las etapas definitivas de este proceso es la cocción, realizada en los hornos. Para su ejecución, el horno pasa por tres etapas: precalentamiento, quema y enfriamiento en el que deben controlarse en forma correcta, con el objeto de obtener productos con mínimos defectos y reducir el impacto ambiental, todo con el máximo rendimiento posible y el mínimo consumo de combustible.

Los ladrillos macizos de arcilla cocidos son utilizados en la construcción de obras civiles principalmente en edificios y viviendas, por los bajos costos del material, su propiedad de aislamiento térmico y su disponibilidad geográfica (Rozo, 2014), la importancia de la cerámica además de lo económico radica en el hecho, que durante la cocción de la pasta moldeada previamente se genera una modificación fundamental en sus propiedades, dando lugar a un material duro de consistencia pétrea que no permite modificar su forma, con alta dureza y resistencia mecánica, resistente al agua y en algunos casos con la capacidad de aislar térmicamente la edificación (Fernández, 2000) .

Sin lugar a dudas, es la cocción la etapa más importante del proceso de fabricación y donde se manifiesta si las etapas de fabricación anteriores se han realizado convenientemente y si el producto cocido ha adquirido las propiedades $y$ características deseadas fijadas por las normas de las Asociaciones de ingeniería sísmica.

La cocción es un proceso físico-químico donde se genera movilidad atómica que une las partículas de arcilla y disminuye la porosidad, es necesario, controlar la velocidad de cocción ya que una contracción rápida puede llevar a tensiones y provocar la rotura por lo cual, es importante considerar el intervalo de cocción, es decir, el rango de temperatura entre el inicio de la vitrificación y el inicio de la deformación, ya que de este intervalo depende de las características de la pasta (Aranguren, 2011).

A nivel nacional la Unidad de Planeación Minera Energética cuenta con el proyecto de orientación del uso eficiente de la energía y de diagnóstico energético, en el que se establece que la industria ladrillera Colombiana tiene un atraso con respecto a estándares internacionales desde los principios de los años 90 debido a la demanda en la construcción, las empresas industriales tuvieron que tecnificar sus procesos con el fin de aumentar la calidad del producto, producción y disminuir costos a través de sistemas tecnificados de producción como uso de máquinas de extrusión de alta calidad y eficiente, implementación de secaderos naturales por artificiales, hornos más eficientes, los cuales consumen menos energía y aumentan la capacidad de producción (Ministerio de Minas y Energía Rozo, 2015). También gestiona buenas prácticas orientadas al uso eficiente de la energía en la industria de cerámicos. En el caso de los hornos, se presentan pautas que se deben tener en cuenta como son: controlar la temperatura de operación en los hornos de acuerdo a lo requerido por los procesos; programar la operación de los hornos a fin de minimizar la frecuencia de puesta en marcha y parada; regular el aire-combustible de quemadores en forma periódica; reparar y reforzar el aislamiento de las paredes del horno; y usar el calor residual para calentar el aire de combustión o proporcionar calor al proceso productivo.

Hoy en día las tecnologías inalámbricas han adoptado en el transcurso del tiempo una manera más sencilla y cómoda de utilizar toda clase de dispositivos, con el fin de mejorar los procesos y/ó las aplicaciones en general. Por ejemplo: Para la telefonía móvil, informática casera, sistemas de seguridad, controles de iluminación, automatización de viviendas (Domótica), aparatos domésticos, alarmas de Incendio/CO2, 
monitorización de sistemas remotos, adquisición de datos de un sensor en sistemas embebidos, etc. (Duran, 2012).

La seguridad en sistemas informáticos es un tópico de alta importancia hoy en día, debido a que nuestros sistemas de información están interconectados con otros sistemas a través de internet o redes propietarias(Parra, 2012), para el proceso de captura y almacenamiento de datos fue necesario contar con una base de datos alojada en un equipo que hizo posible visualizar y controlar los procesos que intervienen entre los que se encuentran; presentación de gráficas en tiempo real y configuración de parámetros de funcionamiento (Sandoval, 2010).

Los perfiles de temperaturas son esenciales para el análisis de la transferencia de energía calorífica, además son de vital importancia para identificar las causas reales de las fallas presentes en los procesos de elaboración de ladrillos y tejas; por esta razón se realizó una herramienta de aplicación, la cual permite la determinación y registro de los perfiles de temperatura con la finalidad de mejorar el funcionamiento de estos hornos.

Estas graficas del proceso de cocción proporcionan parámetros para que el operario modifique la forma en la que se realiza el proceso de transferencia de energía, establezca las condiciones más apropiadas para operar el horno e identifique una ubicación representativa para el monitoreo de temperatura.

Con este instrumento se coloca a disposición las tecnologías virtuales desde la perspectiva de la adquisición de datos, de manera fácil e interactiva para el usuario final.

\section{METODOS}

El estudio se realizó en un horno circular a cielo abierto de la ladrillera el recreo ubicada en el municipio de Ocaña, departamento Norte de Santander, Colombia, a una altitud de 1.227 metros sobre el nivel del mar, bajo las coordenadas $\mathrm{N}$ 1073980 y W 1402088 con una temperatura promedio de $21^{\circ} \mathrm{C}$. El horno de sección transversal circular tiene un diámetro interior de 2,12 metros y una altura de 4,52 metros con espesor de pared de 0,24 metros; la cantidad de ladrillos por quema es de 4.300 y tiene un consumo de carbón de $1.500 \mathrm{~kg}$ por cada cocción, (Ver figura 1).

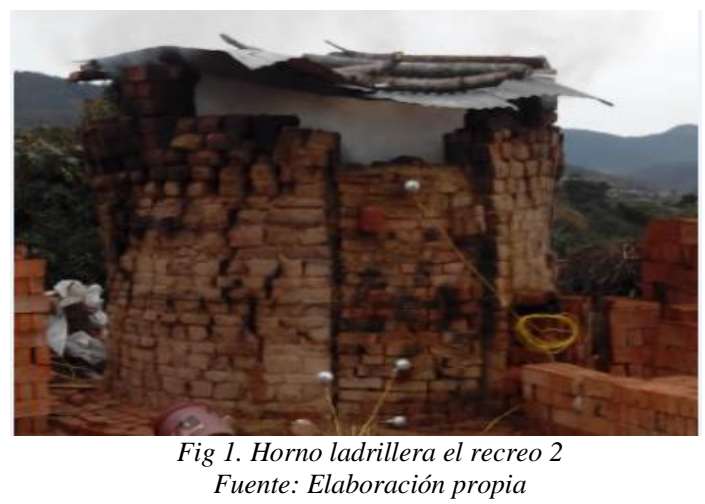

Se diseñó y programó un sistema de adquisición de datos de temperatura, partir de un ejecutable del software manejador DAQ assistant del sistema embebido compact RIO,el cual es el encargado de recibir, procesar y entregar la información ( Ferrín,2013), con una tarjeta de adquisición E/S NI 9213 ensamblada al Chasis NI cDAQ-9184 soportadas por National instruments, permitiendo el procesamiento analógico-digital de la información recibida de los termopares instalados, para luego ser almacenadas a través del software Lab view en el reporte de adquisición y generar los perfiles de temperatura.ver figura 2 .

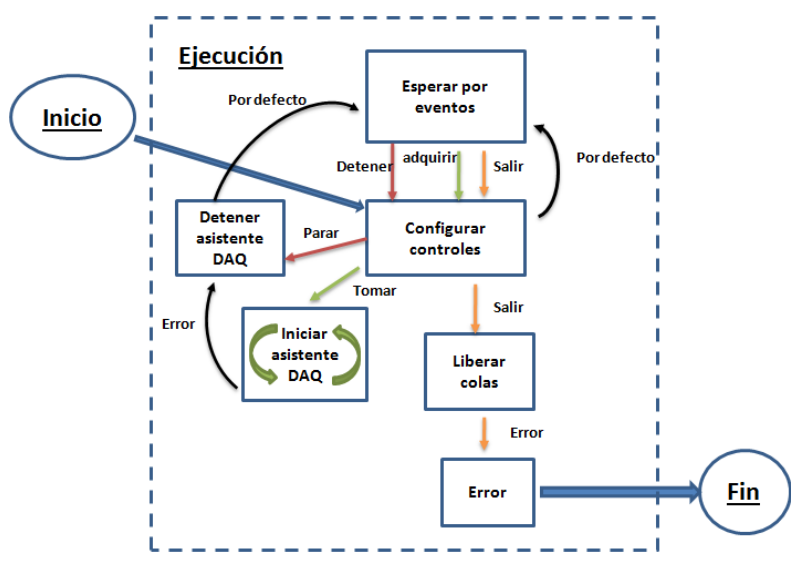

Fig 2. Diagrama de estado para la adquisición de datos en el instrumento

D. E. Marrugo C

Los equipos de adquisición de temperatura, se colocaron en un cuarto de monitoreo ubicado a una distancia cercana al horno seleccionado para la adquisición a través del cable de conexión.

Para el testeo, configuración, visualización y reporte de los datos de adquisición se programó y diseñó el panel frontal utilizando unas pantallas de bienvenida, inicio, testeo del hardware de adquisición, visualización, configuración, reporte, pantalla secundaria de visualización bloque 1 y 
pantalla secundaria de visualización bloque 2 , (Ver figura 3).

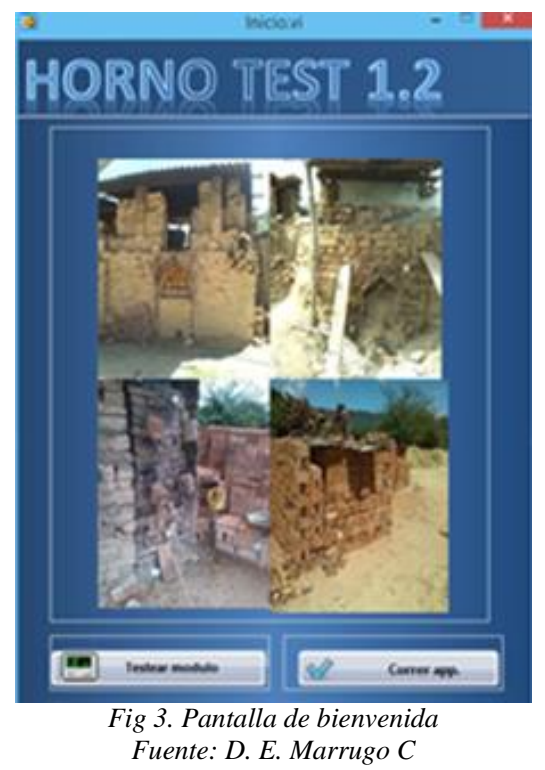

En la ventana de reporte se muestra la barra de estado, de progreso y control de visualizaron de las gráficas de temperaturas individuales y las gráficas por bloque. El programa diseñado, muestra en la pantalla de monitoreo como se van realizando los perfiles de temperatura en tiempo real de las posiciones donde se ubicaron los termopares, (Ver figura 4).

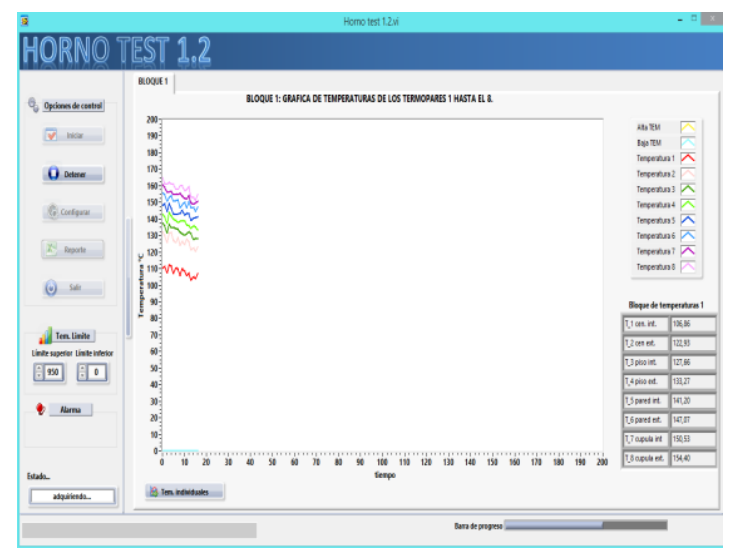

Fig 4. Monitor de temperaturas del instrumento virtual de la adquisición

Fuente: D. E. Marrugo C

La ventana de configuración permite seleccionar el número de termopares a censar y el intervalo de tiempo o "delay" entre cada toma de temperatura y el informe de adquisición se genera en un archivo tipo .xlsx (Microsoft Excel).
Para la selección de los equipos se consideró el bajo costo de los mismos y su amplia utilización como soluciones inalámbricas con ciertas ventajas ya que mejoran el rendimiento del sistema en cuanto a perdida de datos e interferencia y finalmente maximiza el uso del canal al momento de la transmisión de datos (Solarte,2010).

Los termopares utilizados para la medición de temperatura se montan en posiciones representativas para realizar posteriormente la comparación de las curvas de cocción real con la curva dilatométrica ideal. Para registrar las temperaturas presentadas en el proceso de cocción de la arcilla, se instalaron termopares de bulbo de aleación de cromo aluminio tipo $\mathrm{K}$ con aislamiento cerámico.

Los equipos de adquisición de temperatura en el horno se instalan en la casa aledaña al horno a una distancia de $9 \mathrm{~m}$ de la puerta de cargue y descargue de productos, los cuales se instalaron simultáneamente con el proceso de cargue de ladrillos en el horno, (Ver figura 5).

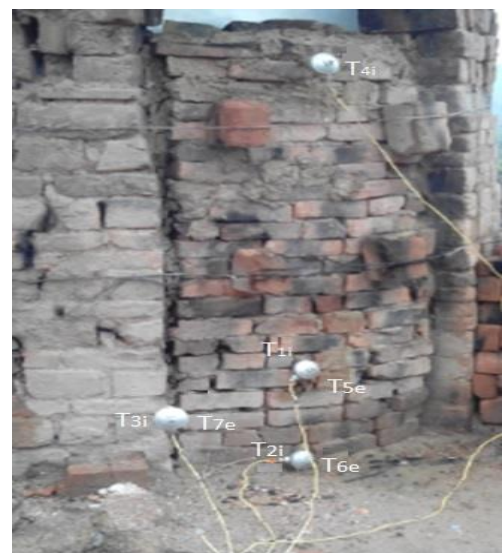

Fig. 5. Ubicación de los termopares utilizados en el horno. Fuente: Elaboración propia

La analogía del proceso de cocción debe comenzar con el establecimiento de la curva ideal de temperaturas, que permita evitar las roturas de precalentamiento, cocción o enfriamiento. Estas roturas, lo mismo que sucede con las de secado, son producidas por tensiones de tracción derivadas de las diferencias de contracción o dilatación que tienen lugar en la misma pieza. Estas diferencias, dependen a su vez, de los gradientes térmicos que en un momento determinado puedan existir en la pieza, los cuales varían a lo largo del proceso de cocción en función de las reacciones endotérmicas o exotérmicas que se producen o de la mayor o 
menor difusividad térmica del material ( Fernández, 2000) .

La pieza responde a las tensiones de cocción deformándose elásticamente. La deformación elástica tiene un límite que se puede llegar a sobrepasar si el calentamiento o enfriamiento son muy rápidos. Entonces la pieza queda sometida a tensiones. Si dichas tensiones son superiores a la resistencia que el material es capaz de oponer a las mismas, se producirán grietas de cocción. Durante la cocción, la arcilla sufre profundas transformaciones que afectan tanto a su elasticidad como a su resistencia mecánica .

Se procede al registro de datos en el horno, utilizando un computador portátil con el desarrollo de la aplicación desarrollada y los driver para la comunicación del sistema embebido compact RIO. Una vez finalizado el montaje de los termopares, se conectaron los terminales de estos al módulo E/S NI 9213 acoplado al chasis NI 9184, y se procede a testear y a ejecutar el programa dando inicio a la adquisición de datos de temperatura. Se programó el software de adquisición para que registrará temperaturas en el intervalo de tiempo cada 5 minutos.

\section{RESULTADOS}

El proceso de registro de los datos inició cuando empezó el proceso combustión del carbón en la parte inferior del horno y terminó hasta el final del proceso de cocción. El monitoreo tuvo una duración de dos días con diez y nueve horas.

El perfil de temperatura generado para la arcilla se muestra en la figura 6 .

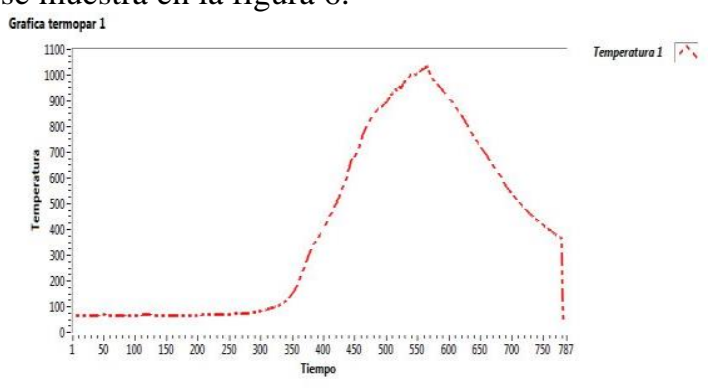

Fig 6. Perfil de temperatura de la arcilla.

Fuente: D. E. Marrugo C
Se realizó la comparación de la curva teórica de temperaturas establecida por Munier, en Chaleur et Industrie al utilizar un análisis termicodilatométrico a la arcilla con la curva de cocción real de la arcilla obtenida en la adquisición de temperatura, (ver figura 7).

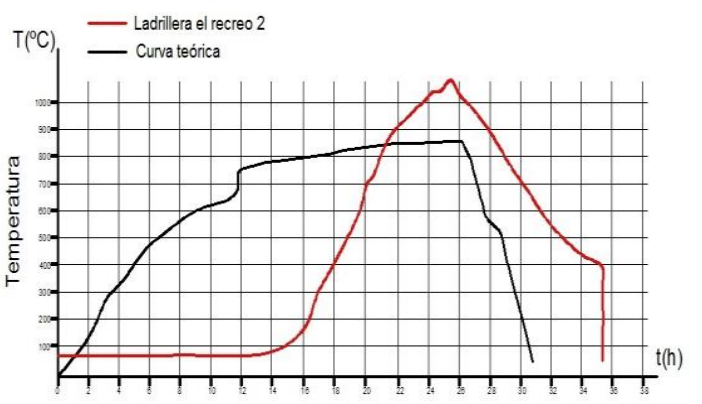

Fig 7. Curva de cocción real y teórica de la cocción de la arcilla.

Fuente: Elaboración propia

\section{RECOMENDACIONES}

Las ladrilleras a cielo abierto debido a que no cuentan con un control y ajuste del proceso de combustión por lo que se presenta un exceso de aire generan un aumento en el consumo de combustible y pérdidas térmicas en el horno, esto es posible de mitigar implementando un sistema de inyección de aire para el proceso de cocción por medio de un ventilador de tiro inducido que permitan mejorar el proceso de combustión, ya que disminuiría las emisiones emitidas al ambiente, se reduciría la cantidad de combustible empleado, se homogenizaría todo el calor en el interior del horno y se mejoraría la calidad de sus productos además no cuentan con sistemas de monitoreo continuo en el proceso de cocción a través de sensores de adquisición y monitoreo a través de termopares en diferentes posiciones en el horno que permitan el registro y/o visualización de datos temperatura de cocción que mejoren las condiciones de dosificación manual, así como registrar curvas típicas para cada uno de los productos, niveles de cocción y coloración deseada. Esta medida permitiría adicionalmente rastrear problemas y dificultades particulares del horno en cada producto. Es necesario reducir las infiltraciones de aire en el horno mediante la aplicación de un estuco en las superficies exteriores ya que este presenta fallas estructurales. 


\section{CONCLUSIONES}

El sistema de adquisición de datos utilizando el software Labview demostró ser una herramienta muy útil, versátil y confiable en el análisis del comportamiento de la cocción de la arcilla en el horno de producción de material cerámico que puede ser utilizado para predecir la influencia de un número de variables en el proceso de cocción. Más allá de la comparación con la curva de cocción de la arcilla, el sistema de adquisición puede obtener diferentes temperaturas de carga y en diferentes posiciones en el horno.

El diseño y la creación de la interfaz gráfica permitió la realización de la adquisición de temperatura en el proceso de cocción y enfriamiento de la arcilla, evidenciando el potencial de la herramienta computacional, con el fin de lograr una reducción en el consumo de energía, como el mejor conocimiento del proceso de cocción en su conjunto y la búsqueda de una mejor calidad en los productos.

Existe un gran desfase durante las primeras horas del precalentamiento entre la curva de cocción real obtenida para la cocción de la arcilla y la curva teórica.

Al inicio en el periodo de humeo se observa que la pasta no alcanzo $\operatorname{los} 400^{\circ} \mathrm{C}$ a las 6 horas de haber comenzado la cocción. Las mediciones efectuadas no daban más de $120^{\circ} \mathrm{C}$. Esto significa, que se estaba efectuando un calentamiento muy lento, precisamente en una zona en que el peligro de roturas es mínimo. Estas 6 primeras horas de cocción pueden considerarse mal aprovechadas.

La fase de humeo se extendió en el horno y en efecto hubo un aumento en la propagación de vapor de agua y en la dilatación de la pasta y se liberaron la mayor cantidad humos, el agua higroscópica es extraída del ladrillo, lo que representa un periodo de dilatación por acumulación de vapor en la pieza.

En las 6 horas siguientes se produce la fase descomposición de la materia orgánica. En esta se elimina el agua combinada, se aumenta la porosidad y se retracta la pasta. Se aprecia en la curva en el horno de la ladrillera una baja velocidad de calentamiento, en cambio, en cambio en la curva teórica, sucede todo lo contrario. Es decir que se acelera dicha velocidad.

El desfase entre la curva en el horno de la ladrillera y la curva teórica, es mucho más acentuado. Dicho desfase, puede dio lugar a una auténtica lluvia ácida sobre el material seco estacionado en la zona de entrada, ahuecando toda su estructura y manchando las superficies expuestas a los gases .

La temperatura máxima en el horno de la ladrillera fue de $1.050^{\circ} \mathrm{C}$, en donde la fase de maduración de la pasta se completa. En este momento la pasta empieza a vitrificarse lo que aumentará la resistentica mecánica a la pieza.

El enfriamiento en el horno de la ladrillera es más lento que el de la curva teórica, soportando sin problemas las tensiones producidas por un enfriamiento rápido.

La pieza finalmente cocida empieza a enfriarse disminuyendo su temperatura paulatinamente por la pérdida de energía transferida hacia las paredes del horno y el ambiente.

La diferencia en la coloración de los productos es un defecto que se observa y que es un factor determinante en la calidad de los productos, esta se da principalmente por la falta de uniformidad de la temperatura dentro del horno, lo que provoca que los materiales que soportan mayores temperaturas tienden a oscurecer el tono rojizo dado por los óxidos de hierro. Otro defecto que se evidenció en la industria cerámica en el municipio de Ocaña son los agrietamientos en los productos estos se deben a la falta de control en la cocción.

Se puede observar que a pesar de la alta tasa de calentamiento y la quema, con velocidades de enfriamiento moderado no causaron graves daños a los ladrillos, como roturas debidas a los choques térmicos.

El tipo de arcilla elegido no era óptima, ya que no se observaron las reacciones de fusión de los componentes con el consiguiente cierre de los poros con la absorción reducida, porque el material es muy arenoso. 


\section{REFERENCIAS}

Asociaciones de ingeniería sísmica. (2004). Guía de Patologías constructivas, estructurales y no estructurales. Santafé de Bogotá.

Aranguren, A., y Sancho, J. (2011). Método de Caracterización de las Propiedades mecánicas de la Fractura del Ladrillo. pp.37 -41. San José de Cúcuta.

Durán,M \& Iturriago,A. (2012). Automatización de un sistema de suministro de agua potable a través de la tecnología zigbee. Revista Colombiana de Tecnologías de Avanzada, 2(20), pp. 36-42.

Fernández Abajo, M. (2000). Manual sobre baldosas, tejas y ladrillos, laboratorio Técnico Cerámico, pp.317 -335, España.

Ferrin,C. \& Magdalena, X. (2013). Sistema de extracción automática de parámetros morfológicos de la huella plantar mediante técnicas de visión por computador en un sistema embebido. Revista colombiana de tecnologías de avanzada, 1(23), pp 8086.

Marrugo D. (2015). Desarrollo de un Instrumento Virtual para la Generación de perfies de Temperatura en Hornos. Revista INGENIO UFPSO, 8 (Ene-Jun), pp 47-58.

Parra, C. \& Herrera. J. (2012). Aplicación de los sistemas de detección de intrusos y la tecnología de agentes en el monitoreo inteligente de redes de datos. Revista colombiana de tecnologías de avanzada, 2 (22), pp 106-110.

Sandoval, G. \& Tobar ,J. (2011). Pluviógrafo electrónico con transmisión de datos inalámbrica. Revista colombiana de tecnologías de avanzada, 1(17), pp. 6773.

Solarte, P. \& Agredo, G. (2013). Diseño de un sistema de alerta temprana para detectar eventos tsunamigénicos asociados con la variación extrema del nivel de la marea en la costa pacífica colombiana. Revista colombiana de tecnologías de avanzada, 1(17), pp. 40-47.

Unidad de Planeación Minero Energética. (2012). Uso eficiente de la energia y de diagnóstico energético, Santafé de Bogotá.

Rozo, S., \& Sánchez, J. A. (2014). Propiedades físico mecánicas de bloques H10 fabricados en el área metropolitana de Cúcuta. Revista
Ciencia e ingeniería Neogranadina, 24(1) pp. 67-78.

\section{SITIOS WEB}

Ministerio de minas y energía, «http://www.minem.gob.pe/minem/archivos/Gu ia12\%20Cer\%C3\%83\%C2\%A1 micos.pdf,». (Consultado 15 febrero 2015). 